\title{
Philosophiques
}

\section{Jean-Paul Desbiens, L'actuel et l'actualité. Sainte-Foy, Le Griffon d'argile, 1986 (Collection Philosophie), 438 pp.}

\section{Philippe Ranger}

Volume 14, numéro 2, automne 1987

URI : https://id.erudit.org/iderudit/027028ar

DOI : https://doi.org/10.7202/027028ar

Aller au sommaire du numéro

Éditeur(s)

Société de philosophie du Québec

ISSN

0316-2923 (imprimé)

1492-1391 (numérique)

Découvrir la revue

Citer ce compte rendu

Ranger, P. (1987). Compte rendu de [Jean-Paul Desbiens, L'actuel et l'actualité. Sainte-Foy, Le Griffon d'argile, 1986 (Collection Philosophie), 438 pp.]

Philosophiques, 14(2), 451-454. https://doi.org/10.7202/027028ar d'utilisation que vous pouvez consulter en ligne.

https://apropos.erudit.org/fr/usagers/politique-dutilisation/ 
JEAN-PAUl Desbiens, L'actuel et l'actualité. Sainte-Foy, Le Griffon d'argile, 1986 (Collection Philosophie), 438 pp.

$$
\text { par Philippe Ranger }
$$

Ce qui vaut à ce volume une recension dans une revue de philosophie, c'est d'abord le titre de la collection où il est publié, ainsi que l'avis liminaire ajouté par son éditeur, Gaston Beaudoin

En publiant ces textes, Le Griffon d'argile reconnaît l'existence d'un public intéressé à une réflexion philosophico-humaniste opposée au discours "langue de bois», produit synthétique sans saveur, issu des idéologies à la mode.

On comprend cet avis de présenter aussi l'auteur comme « penseur libre, non inféodé à des intérêts particuliers ».

Le volume, 438 pages, est le recueil de chroniques parues en 1973 et 1974 dans $A$ Propos, de quelques textes épars, essentiellement de 1974 ou 1983, et surtout de l'ensemble, ou peu s'en faut, des chroniques de l'auteur 
parues de janvier 1984 à juin 1986 dans La Presse (hebdomadairement, durant l'année scolaire). Les 150 textes sont regroupés selon dix sujets.

Le frère Desbiens fut professeur de philosophie avant la Révolution tranquille, puis de 1964 à 1970 responsable au ministère de l'Éducation, où il fit beaucoup pour les cours communs et obligatoires de philosophie dans les cégeps. De 1970 à 1973, il fut éditorialiste en chef à La Presse et, depuis, administrateur de collège privé. Placerait-il lui-même son recueil sous la rubrique « philosophie " ? Son titre et son avant-propos laissent croire qu'il choisirait plutôt « journalisme ». Mais le fait est que ce n'est pas l'opinion de son éditeur. Et cela est un fait intéressant. Nous y reviendrons. Mais voyons pour l'immédiat la question de l'inféodation.

Il est parfaitement vrai que les textes que voici ne sont pas issus d'une iđéologie à la mode, si l'on omet la principale idéologie à la mode depuis une décennie, et qui domine les pages éditoriales de La Presse depuis 1970. Or, quand une mode idéologique domine si longtemps, elle ne fait plus la mode, mais l'état des choses. Et il est vrai sans restriction que ces textes ne sont pas le produit d'une langue de bois - je crois bien que le frère Desbiens n'aurait pas pu écrire le petit éloge que son éditeur veut lui faire.

Il est vrai d'autre part que monsieur Desbiens n'a pas été invité à $L a$ Presse comme journaliste mais comme idéologue. Il est vrai aussi que, si la première citation du volume est de Verlaine, la deuxième est de Léon Bloy, et que les auteurs cités sont le plus souvent Bloy et surtout Alain, pour les inactuels, et ceux de chez Grasset, pour les actuels. Il est vrai aussi que le dernier texte du recueil, un éloge du Dictionnaire canadien des relations de travail de l'abbé Gérard Dion se termine par :

vaut-il la peine de noter que cette ceuvre a été faite gratuitement, comme débordement d'une activité intellectuelle, ignorant les normes, les dispositions négociées, bref, la médiocrité syndiquée.

(Signalons, pour être clair, que c'est M. Desbiens qui maintient au professeur Dion son titre d'abbé, et que je n'entends pas critiquer les deux derniers mots: comment être un syndicat sans syndiquer, au sens strict, la médiocrité?)

Néanmoins, à mon avis l'auteur de ces textes n'est pas inféodé. Il ne livre pas allégeance à un seigneur auquel il pourrait la refuser. Mieux, son succès comme idéologue tient beaucoup à l'effort qu'il met depuis trente ans à ne pas livrer allégeance, ni par l'idée, ni par le style. À veiller à ce que, traitant de sujets sur lesquels on l'interroge, il réponde toujours avec la conviction que le cours de sa pensée comme celui de sa phrase viennent de lui et non des attentes de ceux qui l'écoutent.

Non, s'il faut conserver la métaphore médiévale, ce n'est pas d'inféodation qu'on peut soupçonner l'auteur, mais d'asservissement. Je crois même que, en prenant le mot par le bon côté, le frère Desbiens me suivrait là-dessus. Il attribue (p. 219) à saint François d'Assise le perinde ac cadaver, qu'il interprète comme : 
La pomme ne décide pas d'être pomme; ni le rossignol, rossignol ; ni le saint, un saint.

Je suis sûr que $M$. Desbiens n'a pas plus décidé d'être le personnage qu'il est et que, si c'est pas obéissance habituelle qu'il l'est devenu, cela pour lui ne change rien à l'affaire.

Mais, assez de réflexions sur des textes qui ne sont pas de philosophie. Venons-en au fait qu'ils sont néanmoins inscrits sous cette rubrique. Il faut d'abord reconnaître que le statut de la philosophie dans la cité est tel que cette inscription n'y déroge nullement, et qu'il vaut mieux Jean-Paul Desbiens que Jacques Languirand ou les séries métapsymachinchose qui peuplent la section "philosophie» des librairies encore dépourvues de sections «ésotérisme», "yoga» et "psy». Mais, pour être honnête, il faudrait admettre beaucoup plus.

La femme de la rue bien informée lit-elle des philosophes? À son avis? Ou, si on lui parle de philosophie, à quelle figure favorable peut-elle rattacher le terme, parmi les auteurs qu'elle connaît? Si elle a moins de quarante ans, ou guère plus?

Soyons francs. Nous prions qu'elle vise plus juste, un peu, que son libraire. Et nous n'aurons pas été ignorés des dieux si elle nous répond: le frère Desbiens. Dix ans après la sortie du cégep, le philosophe pour chacun n'est plus Lévy-Strauss ou Krishnamurti, ni Sartre, Nietzsche ou Althusser. Au mieux, le philosophe auquel on prête attention est un maître à penser qui, surtout, ne se pose pas comme tel. Soit : l'idéologue d'une idéologie qui à force de dominer a fini par faire, quant à soi, l'état des choses.

Ces aveux étant faits, nous pouvons nous interroger sur la position rhétorique au moyen de laquelle monsieur Desbiens assume ce statut. C'est la même qui a valu à monsieur Glucksman, par exemple, une certaine mode. La différence ici entre Glucksman et Desbiens est que le premier adoptait cette position par inféodation, ou si vous voulez par méthode, tandis que le second l'adopte le plus sincèrement possible, et depuis toujours, c'est-à-dire depuis qu'il parut sur la place publique sous les traits du frère Untel. Il s'agit bien sûr de la position définie par: "Les autorités nous serinent le contraire, mais l'individu libre doit prendre parole pour dire que... », les trois points tenant lieu d'un aspect de l'état des choses. Cette position fait donc jouer l'autorité fondamentale, assumée par chaque individu libre, contre une autorité éphémère, dépassée ou, simplement, supposée pour l'occasion.

Deux choses étonnent au sujet de cette position. Il s'agit bien de la position typique du maître à penser aujourd'hui. Et elle s'assume avec une merveilleuse indépendance par rapport aux faits. On l'a vu dans la citation au sujet de monsieur Dion, qui laisserait croire que les publications savantes ne font pas partie de la charge de professeur à l'université Laval. On le voit cent autres fois dans le présent recueil. Par exemple, page 97, dans un des éloges dédiés à la revue L'Analyste, à laquelle l'auteur collabore. L'Analyste doit bien 
être la seule revue du genre au Québec à vivre de ses annonceurs. Un autre de ses collaborateurs, deux ans après cette chronique, est devenu directeur du Devoir, sans autre expérience journalistique. Or, l'éloge de la revue se présente comme une réponse à une attaque de Radio Canada, résumée et réfutée en ces termes:

Ainsi donc, L'Analyste serait de droite, et ses partisans sont paranoïaques lorsqu'ils se plaignent de la conspiration du silence qui l'entoure.

Il faut un sacré front pour affirmer... que la presse écrite et électronique n'est qu'un reflet; que les facultés universitaires ou les départements de cégep sont d'une objectivité chimiquement pure.

Le frère Desbiens peut même pousser cela jusqu’au cabotinage, v.g. : «Le Frère provincial des enseignants du Québec, M. Yvon Charbonneau,... » (p. 14). Mais la coupure entre la position rhétorique du maître à penser et les faits ne fut jamais mieux illustrée qu'à l'automne 1970, lorsque M. Desbiens, éditorialiste en chef à $L a$ Presse, critiquant le FLQ et appuyant toutes les mesures gouvernementales, se gagna du fait même la figure de défenseur solitaire et courageux de la vérité et du Québec véritable.

Cette coupure devrait nous éclairer. Ce qui importe ici, ce n'est pas le fond mais strictement la forme. Si le maître à penser naît de la figure de l'individu libre disant ce qui est, contre le discours « inféodé », c'est que cette figure, je crois, assure à chaque spectateur le droit de penser, au sens de penser: plus précisément, qu'elle lui est nécessaire pour actualiser ce droit. La première vertu du maître à penser, c'est cette révélation du droit, de l'obligation de penser. "En quoi il n'est pas vraisemblable que tous se trompent ", mais bien que l'école sous toutes ses formes met en doute le pouvoir de chacun de penser quant à soi.

Il faut donc remercier les dieux que, 350 ans après le Discours, le rôle du «philosophe » pour un certain nombre soit joué par un Desbiens plutôt que par un Glucksman, un Jean Francueur ou un Pierre Pascau. Et peut-être se rappeler que, s'il faut un Desbiens pour assumer si proprement le rôle, il faut d'abord pour le justifier les milliers d'artisans d'une rhétorique d'appareil à laquelle nous croyons aussi sincèrement et parfois aussi librement que M. Desbiens croit à la sienne.

Faculté des sciences de l'éducation

Université de Montréal 